\title{
Regularity results for nonlocal evolution Venttsel' problems
}

\author{
Simone Creo, Maria Rosaria Lancia*, Alexander Nazarov**
}

\begin{abstract}
We consider parabolic nonlocal Venttsel' problems in polygonal and piecewise smooth two-dimensional domains and study existence, uniqueness and regularity in (anisotropic) weighted Sobolev spaces of the solution.
\end{abstract}

Keywords: Venttsel' problems, nonlocal operators, anisotropic weighted Sobolev spaces, piecewise smooth domains.

AMS Subject Classification: 35K20, 35B65. Secondary: 35R02, 35B45.

\section{Introduction}

The aim of this paper is to study the heat equation with nonlocal Venttsel' boundary conditions in a bounded polygonal domain $\Omega \subset \mathbb{R}^{2}$. In the cornerstone paper of Venttsel' [30], a nonlocal term already appears; only recently, many papers deal with nonlocal Venttsel' problems both in the case of smooth and irregular domains. Among the others, we refer to [21], [29], [32], [22] and the references listed in. In this paper, we consider a nonlocal term which can be regarded as a suitable version of the regional fractional Laplacian $(-\Delta)^{s}$, for $s \in(0,1)$, see [7] for applications.

Actually, Venttsel' problems in irregular domains (in particular, domains with pre-fractal or fractal boundary) have been widely investigated, see, e.g., [23, 20], where the reader can

*Dipartimento di Scienze di Base e Applicate per l'Ingegneria, Sapienza Università di Roma, Via A. Scarpa 16, 00161 Roma, Italy. E-mail: simone.creo@sbai.uniroma1.it, mariarosaria.lancia@sbai.uniroma1.it

**St. Petersburg Department of Steklov Mathematical Institute, Fontanka 27, 191023 St. Petersburg, Russia, and St. Petersburg State University, Universitetskii pr. 28, 198504 St. Petersburg, Russia. E-mail: al.il.nazarov@gmail.com 
find also the motivations. We refer, for local linear and quasi-linear Venttsel' problems, to [1], [2], [3], [25], [5], [14], [31], [28], [11], [13], [12], [4] and the references listed in.

In this paper, our goal is to prove regularity results in weighted Sobolev spaces for the weak solution of the problem at hand, thus extending the results obtained in [10] for the elliptic case. When considering the numerical approximation of this problem, to prove regularity results is a key issue for obtaining optimal a priori error estimates. To this regard, see $[8,9]$ for the local case, and [7] for the nonlocal case, under stronger assumptions on the data.

As in the elliptic case [10], it is crucial to prove that the weak solution of the nonlocal Venttsel' problem belongs for a.e. $t$ to the space $H^{2}(\partial \Omega)$; this is achieved by the so-called Munchhausen trick, see, e.g., [10], [4]. To this aim, we introduce suitable anisotropic weighted Sobolev spaces of Kondrat'ev type, see [16, 17], where the weight is the distance from the set of vertices. The techniques used to prove the regularity on the boundary, in the parabolic case, deeply rely also on sophisticated extension theorem in anisotropic Sobolev spaces.

The paper is organized as follows. In Section 1 we define the domain and the functional spaces appearing in this paper, and state the problem. In Section 2 we prove a crucial a priori estimate for the solution. In Section 3 we give an existence and uniqueness result for weak and strong solutions of the parabolic nonlocal Venttsel' problem. Appendix A contains the extension theorem from the broken surface to the whole space.

\section{Statement of the problem}

Let $\Omega \subset \mathbb{R}^{2}$ be a domain with polygonal boundary $\partial \Omega$ with vertices $V_{j}$, for $j=1, \ldots, N$. Namely, we suppose that $\partial \Omega$ is made by $N \geq 3$ segments $l_{j}$, which form a finite number of angles with opening $\alpha_{j}$, and let us denote with $\alpha$ the opening of the largest angle in $\partial \Omega$, see Figure 1 . We denote by $V(\partial \Omega)$ the set of vertices $V_{j}$.

In the following we denote with $L^{2}(\Omega)$ the Lebesgue space with respect to the Lebesgue measure $d x$ on $\Omega$, and with $L^{2}(\partial \Omega)$ the Lebesgue space on the boundary with respect to the arc length $d \ell$. By $H^{s}(\Omega)$, for $s>0$, we denote the standard Sobolev-Slobodetskii spaces. By $\mathcal{C}(\partial \Omega)$ we denote the set of continuous functions on $\partial \Omega$, and by $\mathcal{C}_{0}^{\infty}(\mathbb{R} \times \mathbb{R})$ we denote the set of infinitely differentiable functions with compact support in $\mathbb{R} \times \mathbb{R}$. Moreover, we denote by $B_{1}(0)$ the unit ball centered in the origin.

By $H^{s}(\partial \Omega)$, for $0<s<1$, we denote the Sobolev-Slobodetskii space on $\partial \Omega$ defined by local Lipschitz charts as in [26]. For $s \geq 1$, we define the space $H^{s}(\partial \Omega)$ by using the 


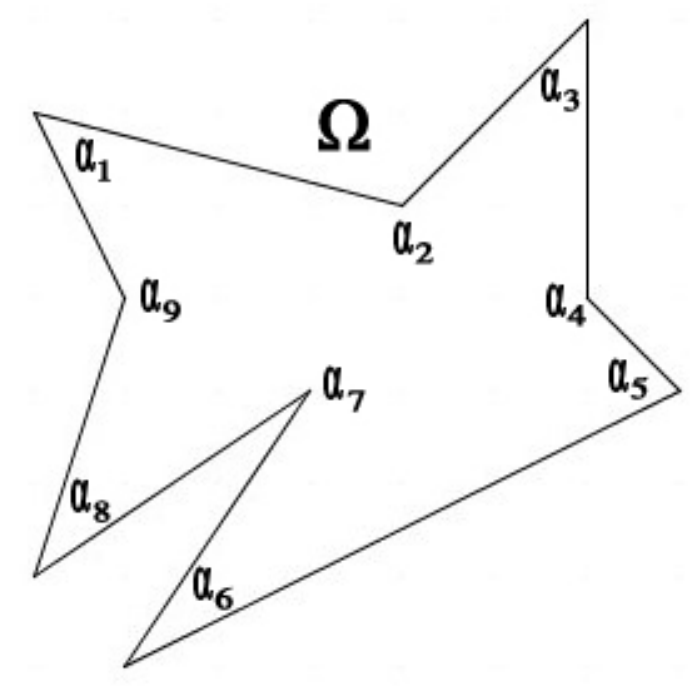

Figure 1: A possible example of domain $\Omega$. In this case $N=9$ and $\alpha=\alpha_{7}$.

characterization given by Brezzi-Gilardi in [6]:

$$
H^{s}(\partial \Omega)=\left\{v \in \mathcal{C}(\partial \Omega):\left.v\right|_{\stackrel{\circ}{M}} \in H^{s}(\stackrel{\circ}{M})\right\}
$$

where $M$ denotes a side of $\partial \Omega$ and $\stackrel{\circ}{M}$ denotes the corresponding open segment (for the general case see Definition 2.27 in [6]).

We fix a counterclockwise orientation on $\partial \Omega$. We denote by $L_{j}$ the length of the segment $l_{j}$, for $j=1, \ldots, N$, and by $L$ the length of $\partial \Omega$.

We choose $V_{1}$ as the origin and we parametrize $\partial \Omega$ by the arc-length

$$
\phi_{1}(\ell)=\phi_{V_{1}}(\ell), \quad \phi_{1}(\ell):[0, L] \rightarrow \mathbb{R}^{2},
$$

with $\phi_{1}$ continuous, injective in $[0, L]$ and such that $\phi_{1}(0)=\phi_{1}(L)$.

By choosing as origin $V_{j}$, we define in a similar way

$$
\phi_{j}(\ell)=\phi_{V_{j}}(\ell), \quad \ell \in[0, L]
$$

For every $j=1, \ldots, N$, we set

$$
\nabla_{\ell}^{+} u\left(V_{j}\right):=\lim _{h \rightarrow 0^{+}} \nabla_{\ell} u\left(\phi_{j}\left(L_{j}+h\right)\right), \quad \nabla_{\ell}^{-} u\left(V_{j}\right):=\lim _{h \rightarrow 0^{-}} \nabla_{\ell} u\left(\phi_{j}\left(L_{j}+h\right)\right),
$$

where $\nabla_{\ell}=\frac{\partial}{\partial \ell}$, and define the subspace

$$
\tilde{H}^{2}(\partial \Omega)=\left\{u \in H^{2}(\partial \Omega): u \circ \phi_{j} \in H^{2}\left(0, L_{j}\right) \text { and } \nabla_{\ell}^{+} u\left(V_{j}\right)=\nabla_{\ell}^{-} u\left(V_{j}\right) \forall j=1, \ldots, N\right\} .
$$


Let $r=r(x)$ be the distance from the set of vertices $V_{j}$. For $\gamma \in \mathbb{R}$, and $m=0,1,2, \ldots$, we denote by $H_{\gamma}^{m}(\Omega)$ the Kondrat'ev space of functions for which the norm

$$
\|u\|_{H_{\gamma}^{m}(\Omega)}=\left(\sum_{|k| \leq m} \int_{\Omega} r^{2(\gamma-m+|k|)}\left|D^{k} u(x)\right|^{2} d x\right)^{\frac{1}{2}}
$$

is finite, see [16]. For $m=0$, this space evidently coincides with the weighted Lebesgue space $L_{\gamma}^{2}(\Omega)$. We also define, for $m \in \mathbb{N}$, the space $H_{\gamma}^{m-\frac{1}{2}}(\partial \Omega)$ as the trace space of $H_{\gamma}^{m}(\Omega)$ equipped with the norm

$$
\|u\|_{H_{\gamma}^{m-\frac{1}{2}}(\partial \Omega)}=\inf _{v=u \text { on } \partial \Omega}\|v\|_{H_{\gamma}^{m}(\Omega)} .
$$

We now introduce anisotropic Sobolev spaces on the cylinder $Q_{T}=\Omega \times(0, T)$ and its lateral surface $\partial^{\prime \prime} Q_{T}=\partial \Omega \times(0, T)$. For $l, m \geq 0$ we define

$$
H^{l, m}\left(Q_{T}\right)=L^{2}\left([0, T] ; H^{l}(\Omega)\right) \cap H^{m}\left([0, T] ; L^{2}(\Omega)\right),
$$

and by $H^{l, m}\left(\partial^{\prime \prime} Q_{T}\right)$ we denote the analogous space on $\partial^{\prime \prime} Q_{T}$, taking into account the previous definition of the space $H^{s}(\partial \Omega)$. Similarly, we define

$$
\tilde{H}^{2,1}\left(\partial^{\prime \prime} Q_{T}\right)=L^{2}\left([0, T] ; \tilde{H}^{2}(\partial \Omega)\right) \cap H^{1}\left([0, T] ; L^{2}(\partial \Omega)\right) .
$$

We introduce also the anisotropic Kondrat'ev space $H_{\gamma}^{2,1}\left(Q_{T}\right)$ of functions for which the following norm is finite (see [17]):

$$
\|u\|_{H_{\gamma}^{2,1}\left(Q_{T}\right)}=\left(\int_{Q_{T}} r^{2(\gamma-2)} \sum_{|\bar{\alpha}| \leq 2} r^{2|\bar{\alpha}|}\left|\partial_{t}^{\alpha_{0}} D_{x}^{\alpha} u\right|^{2} d x d t\right)^{\frac{1}{2}}
$$

where $\bar{\alpha}=\left(\alpha_{0}, \alpha\right)$ and $|\bar{\alpha}|=2 \alpha_{0}+|\alpha|$.

We denote the trace of $u$ on $\partial^{\prime \prime} Q_{T}$ with $\gamma_{0} u$. Sometimes we will use the same symbol $u$ to denote the function itself and its trace $\gamma_{0} u$. The interpretation will be left to the context.

We define the composite spaces

$$
\begin{aligned}
& V^{1,0}\left(Q_{T}, \partial^{\prime \prime} Q_{T}\right):=\left\{u \in H^{1,0}\left(Q_{T}\right): \gamma_{0} u \in H^{1,0}\left(\partial^{\prime \prime} Q_{T}\right)\right\} \\
& V^{1,1}\left(Q_{T}, \partial^{\prime \prime} Q_{T}\right):=\left\{u \in H^{1,1}\left(Q_{T}\right): \gamma_{0} u \in H^{1,1}\left(\partial^{\prime \prime} Q_{T}\right)\right\},
\end{aligned}
$$

and, for $\sigma \in \mathbb{R}$,

$$
V_{\sigma}^{2,1}\left(Q_{T}, \partial^{\prime \prime} Q_{T}\right):=\left\{u \in H^{1,0}\left(Q_{T}\right): r^{\sigma} D^{2} u \in L^{2}\left(Q_{T}\right), r^{\sigma} u_{t} \in L^{2}\left(Q_{T}\right), \gamma_{0} u \in \tilde{H}^{2,1}\left(\partial^{\prime \prime} Q_{T}\right)\right\}
$$


We consider the problem formally stated as

$$
\begin{array}{ll}
u_{t}-\Delta u+a u=f & \text { in } Q_{T}, \\
u_{t}-\Delta_{\ell} u+\frac{\partial u}{\partial \nu}+b u+\theta_{s}(u)=g & \text { on } \partial^{\prime \prime} Q_{T}, \\
u(x, 0)=0 & \text { on } \bar{\Omega},
\end{array}
$$

where $f$ and $g$ are given functions, $\Delta_{\ell}=\frac{\partial^{2}}{\partial \ell^{2}}, \nu$ is the unit vector of exterior normal, $a \in L^{\infty}\left(Q_{T}\right), b \in L^{\infty}\left(\partial^{\prime \prime} Q_{T}\right)$ and, for $s \in(0,1)$, we set $\theta_{s}: H^{s}(\partial \Omega) \rightarrow H^{-s}(\partial \Omega)$ as follows: for every $u, v \in H^{s}(\partial \Omega)$

$$
\left\langle\theta_{s}(u), v\right\rangle=\iint_{\partial \Omega \times \partial \Omega} \frac{(u(x)-u(y))(v(x)-v(y))}{|x-y|^{1+2 s}} d \ell(x) d \ell(y)
$$

where $\langle\cdot, \cdot\rangle$ denotes the duality pairing between $H^{-s}(\partial \Omega)$ and $H^{s}(\partial \Omega)$. We remark that the nonlocal term $\theta_{s}(\cdot)$ can be regarded as an analogue of the regional fractional Laplace operator $(-\Delta)_{\partial \Omega}^{s}$ on $\partial \Omega$.

We now define the bilinear form $E(u, v)$ as follows:

$$
E(u, v)=\int_{\Omega} \nabla u \nabla v d x+\int_{\partial \Omega} \nabla_{\ell} u \nabla_{\ell} v d \ell+\int_{\Omega} a u v d x+\int_{\partial \Omega} b u v d \ell+\left\langle\theta_{s}(u), v\right\rangle,
$$

for every $u, v \in V^{1}(\Omega, \partial \Omega):=\left\{u \in H^{1}(\Omega):\left.u\right|_{\partial \Omega} \in H^{1}(\partial \Omega)\right\}$.

We consider the weak formulation of the problem (1.1)-(1.3) (cf. [24]):

Given $f$ and $g$, find $u \in V^{1,0}\left(Q_{T}, \partial^{\prime \prime} Q_{T}\right)$ such that

$$
-\int_{Q_{T}} u v_{t} d x d t-\int_{\partial^{\prime \prime} Q_{T}} u v_{t} d \ell d t+\int_{0}^{T} E(u, v) d t=\int_{Q_{T}} f v d x d t+\int_{\partial^{\prime \prime} Q_{T}} g v d \ell d t
$$

for every $v \in V^{1,1}\left(Q_{T}, \partial^{\prime \prime} Q_{T}\right)$ such that $v(T, x)=0$.

Proposition 1.1. Let $u$ be a weak solution of (1.1)-(1.3). Suppose that $r^{\sigma} D^{2} u \in L^{2}\left(Q_{T}\right)$, $r^{\sigma} u_{t} \in L^{2}\left(Q_{T}\right)$ and $\gamma_{0} u \in H^{2,1}\left(\partial^{\prime \prime} Q_{T}\right)$. Then $u$ is a strong solution, i.e. equalities (1.1)-(1.3) are satisfied a.e. in $Q_{T}$, on $\partial^{\prime \prime} Q_{T}$ and in $\Omega$, respectively. Moreover, $\gamma_{0} u \in \tilde{H}^{2,1}\left(\partial^{\prime \prime} Q_{T}\right)$, i.e. $u \in V_{\sigma}^{2,1}\left(Q_{T}, \partial^{\prime \prime} Q_{T}\right)$

This statement follows from integration by parts and the fundamental lemma of calculus of variations.

In what follows we denote by $C$ all positive constants. The dependence of constants on some parameters is given in parentheses. We do not indicate the dependence of $C$ on the geometry of $\Omega$. 


\section{A priori estimates}

Theorem 2.1. Let $u \in V_{\sigma}^{2,1}\left(Q_{T}, \partial^{\prime \prime} Q_{T}\right)$ be a solution of problem (1.1)-(1.3). Then there exists a positive constant $C=C(\sigma)$ such that

$$
\begin{aligned}
& \|u\|_{H^{1,0}\left(Q_{T}\right)}^{2}+\left\|r^{\sigma} D^{2} u\right\|_{L^{2}\left(Q_{T}\right)}^{2}+\left\|r^{\sigma} u_{t}\right\|_{L^{2}\left(Q_{T}\right)}^{2}+\|u\|_{H^{2,1}\left(\partial^{\prime \prime} Q_{T}\right)}^{2} \\
& \leq C(\sigma)\left(\|u\|_{L^{2}\left(\partial^{\prime \prime} Q_{T}\right)}^{2}+\left\|r^{\sigma} f\right\|_{L^{2}\left(Q_{T}\right)}^{2}+\|g\|_{L^{2}\left(\partial^{\prime \prime} Q_{T}\right)}^{2}\right)
\end{aligned}
$$

provided

$$
1-\frac{\pi}{\alpha}<\sigma<\frac{1}{2}, \quad \sigma \geq-\frac{1}{2}
$$

(recall that $\alpha$ is the opening of the largest angle in $\partial \Omega$ ).

Proof. We use the Munchhausen trick. We move the terms $\frac{\partial u}{\partial \nu}, b u$ and $\theta_{s}(u)$ in (1.2) into the right-hand side and consider them as known functions. Then we easily have

$$
\|u\|_{H^{2,1}\left(\partial^{\prime \prime} Q_{T}\right)}^{2} \leq C\left(\left\|\frac{\partial u}{\partial \nu}\right\|_{L^{2}\left(\partial^{\prime \prime} Q_{T}\right)}^{2}+\|u\|_{L^{2}\left(\partial^{\prime \prime} Q_{T}\right)}^{2}+\left\|\theta_{s}(u)\right\|_{L^{2}\left(\partial^{\prime \prime} Q_{T}\right)}^{2}+\|g\|_{L^{2}\left(\partial^{\prime \prime} Q_{T}\right)}^{2}\right) .
$$

We proceed in several steps.

1) First we estimate $\left\|\theta_{s}(u)\right\|_{L^{2}\left(\partial^{\prime \prime} Q_{T}\right)}^{2}$. Since $u \in \tilde{H}^{2,1}\left(\partial^{\prime \prime} Q_{T}\right)$, in particular $u(\cdot, t) \in$ $\tilde{H}^{2}(\partial \Omega)$ for a.e. $t$. Hence it is sufficient to consider the local behavior of $u$ near the vertices. Without loss of generality, we can assume that the vertex is located at the origin. We introduce a smooth cutoff function $\eta$ and rectify $\partial \Omega$ near the origin. From our hypothesis on $u$, we have that for a.e. $t \in[0, T] \theta_{s}(u(\cdot, t)) \in H^{2-2 s}(\partial \Omega)$ and

$$
\left\|\theta_{s}(u(\cdot, t))\right\|_{H^{2-2 s}(\partial \Omega)}^{2} \leq C(s)\|u(\cdot, t)\|_{H^{2}(\partial \Omega)}^{2} .
$$

From the compact embedding of $H^{2-2 s}(\partial \Omega)$ in $L^{2}(\partial \Omega)$ we deduce that for every $\varepsilon>0$ there exists a constant $C(\varepsilon)$ such that

$$
\left\|\theta_{s}(u(\cdot, t))\right\|_{L^{2}(\partial \Omega)}^{2} \leq \varepsilon\left\|\theta_{s}(u(\cdot, t))\right\|_{H^{2-2 s}(\partial \Omega)}^{2}+C(\varepsilon)\left\|\theta_{s}(u(\cdot, t))\right\|_{H^{-s}(\partial \Omega)}^{2},
$$

see Lemma 6.1, Chapter 2 in [26]. Similarly, we have

$$
\left\|\theta_{s}(u(\cdot, t))\right\|_{H^{-s}(\partial \Omega)}^{2} \leq C\|u(\cdot, t)\|_{H^{s}(\partial \Omega)}^{2} \leq \varepsilon\|u(\cdot, t)\|_{H^{2}(\partial \Omega)}^{2}+C(\varepsilon)\|u\|_{L^{2}(\partial \Omega)}^{2}
$$

Putting together these estimates, we get

$$
\left\|\theta_{s}(u(\cdot, t))\right\|_{L^{2}(\partial \Omega)}^{2} \leq \varepsilon\|u(\cdot, t)\|_{H^{2}(\partial \Omega)}^{2}+C(\varepsilon)\|u(\cdot, t)\|_{L^{2}(\partial \Omega)}^{2} .
$$


By integrating (2.4) with respect to $t \in[0, T]$ we obtain

$$
\begin{aligned}
\left\|\theta_{s}(u)\right\|_{L^{2}\left(\partial^{\prime \prime} Q_{T}\right)}^{2} & =\int_{0}^{T}\left\|\theta_{s}(u(\cdot, t))\right\|_{L^{2}(\partial \Omega)}^{2} d t \leq \int_{0}^{T}\left(\varepsilon\|u(\cdot, t)\|_{H^{2}(\partial \Omega)}^{2}+C(\varepsilon)\|u(\cdot, t)\|_{L^{2}(\partial \Omega)}^{2}\right) d t \\
& =\varepsilon\|u\|_{L^{2}\left([0, T] ; H^{2}(\partial \Omega)\right)}^{2}+C(\varepsilon)\|u\|_{L^{2}\left(\partial^{\prime \prime} Q_{T}\right)}^{2} \leq \varepsilon\|u\|_{H^{2,1}\left(\partial^{\prime \prime} Q_{T}\right)}^{2}+C(\varepsilon)\|u\|_{L^{2}\left(\partial^{\prime \prime} Q_{T}\right)}^{2} .
\end{aligned}
$$

Therefore we obtain the following estimate using (2.3):

$$
\|u\|_{H^{2,1}\left(\partial^{\prime \prime} Q_{T}\right)}^{2} \leq C\left(\left\|\frac{\partial u}{\partial \nu}\right\|_{L^{2}\left(\partial^{\prime \prime} Q_{T}\right)}^{2}+\|g\|_{L^{2}\left(\partial^{\prime \prime} Q_{T}\right)}^{2}+\varepsilon\|u\|_{H^{2,1}\left(\partial^{\prime \prime} Q_{T}\right)}^{2}+C(\varepsilon)\|u\|_{L^{2}\left(\partial^{\prime \prime} Q_{T}\right)}^{2}\right) .
$$

By choosing $\varepsilon$ sufficiently small we obtain

$$
\|u\|_{H^{2,1}\left(\partial^{\prime \prime} Q_{T}\right)}^{2} \leq C\left(\left\|\frac{\partial u}{\partial \nu}\right\|_{L^{2}\left(\partial^{\prime \prime} Q_{T}\right)}^{2}+\|u\|_{L^{2}\left(\partial^{\prime \prime} Q_{T}\right)}^{2}+\|g\|_{L^{2}\left(\partial^{\prime \prime} Q_{T}\right)}^{2}\right) .
$$

2) By Theorem A.1, there is an extension $U \in H^{\frac{5}{2}, \frac{5}{4}}\left(\mathbb{R}^{2} \times \mathbb{R}\right)$ such that $\left.(U-u)\right|_{\partial^{\prime \prime} Q_{T}}=0$, $\left.U\right|_{t=0}=0$, and the following estimate holds:

$$
\|U\|_{H^{\frac{5}{2}, \frac{5}{4}\left(\mathbb{R}^{2} \times \mathbb{R}\right)}} \leq C\|u\|_{H^{2,1}\left(\partial^{\prime \prime} Q_{T}\right)} .
$$

Without loss of generality we can suppose that the support of $U$ is bounded.

We claim that $D^{2} U$ and $U_{t}$ belong to the weighted Lebesgue space $L_{-\frac{1}{2}}^{2}\left(\mathbb{R}^{2} \times \mathbb{R}\right)$. Indeed, by localizing we need to check it only in a neighborhood of a vertex $V_{j}$ located at the origin.

The inclusion of $U \in H^{\frac{5}{2}, \frac{5}{4}}\left(\mathbb{R}^{2} \times \mathbb{R}\right)$ evidently implies $D^{2} U \in L^{2}\left(\mathbb{R} ; H^{\frac{1}{2}}\left(\mathbb{R}^{2}\right)\right)$. Furthermore, the Young inequality

$$
|\eta||\xi|^{\frac{1}{2}} \leq \frac{|\eta|^{\frac{5}{4}}}{5 / 4}+\frac{|\xi|^{\frac{5}{2}}}{5}
$$

shows that $U_{t} \in L^{2}\left(\mathbb{R} ; H^{\frac{1}{2}}\left(\mathbb{R}^{2}\right)\right)$, and (2.7) gives

$$
\left\|D^{2} U\right\|_{L^{2}\left(\mathbb{R} ; H^{\left.\frac{1}{2}\left(\mathbb{R}^{2}\right)\right)}\right.}+\left\|U_{t}\right\|_{L^{2}\left(\mathbb{R} ; H^{\left.\frac{1}{2}\left(\mathbb{R}^{2}\right)\right)}\right.} \leq C\|u\|_{H^{2,1}\left(\partial^{\prime \prime} Q_{T}\right)}
$$

By the fractional Hardy inequality, see [15, Theorem 3.2 and Remark 3.2], for a.e. $t$ we have

$$
\int_{\mathbb{R}^{2}} \frac{\left|D^{2} U(\cdot, t)\right|^{2}}{|x|} d x \leq C\left\|D^{2} U(\cdot, t)\right\|_{H^{\frac{1}{2}\left(\mathbb{R}^{2}\right)}}^{2}
$$

and a similar inequality holds for $U_{t}$. We integrate these estimates with respect to $t$, and the claim follows.

3) We now consider the function $v=u-U$. It solves the Dirichlet problem

$$
v_{t}-\Delta v=f-U_{t}+\Delta U \in L_{\sigma}^{2}\left(Q_{T}\right) ;\left.\quad v\right|_{\partial^{\prime \prime} Q_{T}}=0 ;\left.\quad v\right|_{t=0}=0
$$


(here we used the last restriction in (2.2)). From Theorem 3 in [18] (with $l=0$ ) it follows that $v \in H_{\sigma}^{2,1}\left(Q_{T}\right)$ if $|\sigma-1|<\frac{\pi}{\alpha}$ (we recall that $\alpha$ is the opening of the largest angle in $\partial \Omega$ ). From (2.8) and (2.9), this implies

$$
\|u\|_{H^{1,0}\left(Q_{T}\right)}^{2}+\left\|r^{\sigma} D^{2} u\right\|_{L^{2}\left(Q_{T}\right)}^{2}+\left\|r^{\sigma} u_{t}\right\|_{L^{2}\left(Q_{T}\right)}^{2} \leq C(\sigma)\left(\left\|r^{\sigma} f\right\|_{L^{2}\left(Q_{T}\right)}^{2}+\|u\|_{H^{2,1}\left(\partial^{\prime \prime} Q_{T}\right)}^{2}\right)
$$

(to estimate the first term, we also take into account that (2.2) implies $\sigma \leq 1$ ).

4) We are now in the position to estimate $\left\|\frac{\partial u}{\partial \nu}\right\|_{L^{2}\left(\partial^{\prime \prime} Q_{T}\right)}^{2}$. By rescaling, we deduce that $\nabla u \in L_{\sigma-\frac{1}{2}}^{2}\left(\partial^{\prime \prime} Q_{T}\right)$ and

$$
\|\nabla u\|_{L_{\sigma-\frac{1}{2}}^{2}\left(\partial^{\prime \prime} Q_{T}\right)}^{2} \leq C\left(\|u\|_{H^{1,0}\left(Q_{T}\right)}^{2}+\left\|r^{\sigma} D^{2} u\right\|_{L^{2}\left(Q_{T}\right)}^{2}\right) .
$$

Following [10], we define a cutoff function $\eta_{\delta}$ such that

$$
\eta_{\delta}(r)=1 \quad \text { for } \quad r>\delta, \quad \eta_{\delta}(r)=0 \quad \text { for } \quad r<\delta / 2
$$

and we introduce the following trace operator:

$$
\left.u \longrightarrow \frac{\partial u}{\partial \nu}\right|_{\partial^{\prime \prime} Q_{T}}=\left.\eta_{\delta} \frac{\partial u}{\partial \nu}\right|_{\partial^{\prime \prime} Q_{T}}+\left.\left(1-\eta_{\delta}\right) \frac{\partial u}{\partial \nu}\right|_{\partial^{\prime \prime} Q_{T}}=: \mathcal{K}_{1}(\delta) u+\mathcal{K}_{2}(\delta) u
$$

The operator $\mathcal{K}_{1}(\delta): H_{\sigma}^{2,1}\left(Q_{T}\right) \rightarrow L^{2}\left(\partial^{\prime \prime} Q_{T}\right)$ is evidently compact. Using (2.11), we obtain for arbitrary $\varepsilon>0$

$$
\left\|\mathcal{K}_{1}(\delta) u\right\|_{L^{2}\left(\partial^{\prime \prime} Q_{T}\right)}^{2} \leq \frac{\varepsilon}{2}\left(\left\|r^{\sigma} f\right\|_{L^{2}\left(Q_{T}\right)}^{2}+\|u\|_{H^{2,1}\left(\partial^{\prime \prime} Q_{T}\right)}^{2}\right)+C(\varepsilon, \sigma, \delta)\|u\|_{L^{2}\left(\partial^{\prime \prime} Q_{T}\right)}^{2} .
$$

From (2.11) and (2.12) we deduce

$$
\left\|\mathcal{K}_{2}(\delta) u\right\|_{L^{2}\left(\partial^{\prime \prime} Q_{T}\right)}^{2} \leq C(\sigma) \delta^{\frac{1}{2}-\sigma}\left(\left\|r^{\sigma} f\right\|_{L^{2}\left(Q_{T}\right)}^{2}+\|u\|_{H^{2,1}\left(\partial^{\prime \prime} Q_{T}\right)}^{2}\right)
$$

By choosing $\delta(\sigma, \varepsilon)$ sufficiently small we get

$$
\left\|\frac{\partial u}{\partial \nu}\right\|_{L^{2}\left(\partial^{\prime \prime} Q_{T}\right)}^{2} \leq \varepsilon\left(\left\|r^{\sigma} f\right\|_{L^{2}\left(Q_{T}\right)}^{2}+\|u\|_{H^{2,1}\left(\partial^{\prime \prime} Q_{T}\right)}^{2}\right)+C(\varepsilon, \sigma)\|u\|_{L^{2}\left(\partial^{\prime \prime} Q_{T}\right)}^{2} .
$$

Substituting the above inequality into (2.6) we have

$$
\|u\|_{H^{2,1}\left(\partial^{\prime \prime} Q_{T}\right)}^{2} \leq C\left(\varepsilon\left(\left\|r^{\sigma} f\right\|_{L^{2}\left(Q_{T}\right)}^{2}+\|u\|_{H^{2,1}\left(\partial^{\prime \prime} Q_{T}\right)}^{2}\right)+C(\varepsilon, \sigma)\|u\|_{L^{2}\left(\partial^{\prime \prime} Q_{T}\right)}^{2}+\|g\|_{L^{2}\left(\partial^{\prime \prime} Q_{T}\right)}^{2}\right) .
$$

By choosing $\varepsilon$ sufficiently small we obtain

$$
\|u\|_{H^{2,1}\left(\partial^{\prime \prime} Q_{T}\right)}^{2} \leq C\left(\left\|r^{\sigma} f\right\|_{L^{2}\left(Q_{T}\right)}^{2}+C(\sigma)\|u\|_{L^{2}\left(\partial^{\prime \prime} Q_{T}\right)}^{2}+\|g\|_{L^{2}\left(\partial^{\prime \prime} Q_{T}\right)}^{2}\right) .
$$

Taking into account (2.11), we get (2.1). 


\section{Strong solvability of the Venttsel' problem}

We begin with the existence and uniqueness of the weak solution. By standard Galerkin methods (cf. [19]), the following result holds.

Lemma 3.1. Let $f \in L^{2}(\Omega), g \in L^{2}(\partial \Omega), a \in L^{\infty}\left(Q_{T}\right)$ and $b \in L^{\infty}\left(\partial^{\prime \prime} Q_{T}\right)$. Then there exists a unique weak solution $u$ in $V^{1,0}\left(Q_{T}, \partial^{\prime \prime} Q_{T}\right)$ of problem (1.5). Moreover

$$
\|u\|_{V^{1,0}\left(Q_{T}, \partial^{\prime \prime} Q_{T}\right)} \leq C\left(\|f\|_{L^{2}\left(Q_{T}\right)}+\|g\|_{L^{2}\left(\partial^{\prime \prime} Q_{T}\right)}\right),
$$

where $C$ depends on $T$, $a$ and $b$.

We finally prove the desired regularity for the weak solution of the parabolic nonlocal Venttsel' problem.

Theorem 3.2. Let $\sigma$ be subject to condition (2.2). Suppose that $g$, a and $b$ are as in Lemma 3.1 and that $f \in L_{\sigma}^{2}(\Omega)$. Then the problem (1.1)-(1.3) has a unique solution $u \in$ $V_{\sigma}^{2,1}\left(Q_{T}, \partial^{\prime \prime} Q_{T}\right)$, and the following inequality holds:

$$
\begin{aligned}
& \|u\|_{H^{1,0}\left(Q_{T}\right)}^{2}+\left\|r^{\sigma} D^{2} u\right\|_{L^{2}\left(Q_{T}\right)}^{2}+\left\|r^{\sigma} u_{t}\right\|_{L^{2}\left(Q_{T}\right)}^{2}+\|u\|_{H^{2,1}\left(\partial^{\prime \prime} Q_{T}\right)}^{2} \\
& \leq C\left(\left\|r^{\sigma} f\right\|_{L^{2}\left(Q_{T}\right)}^{2}+\|g\|_{L^{2}\left(\partial^{\prime \prime} Q_{T}\right)}^{2}\right),
\end{aligned}
$$

where $C$ depends on $\sigma, T$, $a$ and $b$.

Proof. We proceed similarly to the proof of [10, Theorem 3.3]. We introduce the set of operators $\mathcal{L}_{\mu}: V_{\sigma}^{2,1}\left(Q_{T}, \partial^{\prime \prime} Q_{T}\right) \rightarrow L_{\sigma}^{2}\left(Q_{T}\right) \times L^{2}\left(\partial^{\prime \prime} Q_{T}\right)$ as follows:

$$
\mathcal{L}_{\mu} u:=\left(u_{t}-\Delta u+\mu a u,\left.\left(u_{t}-\Delta_{\ell} u+\mu\left(\frac{\partial u}{\partial \nu}+b u+\theta_{s}(u)\right)\right)\right|_{\partial \Omega}\right) .
$$

We claim that the operator $\mathcal{L}_{0}$ is invertible. Indeed, it corresponds to the boundary value problem

$$
u_{t}-\Delta u=f \quad \text { in } Q_{T}, \quad u_{t}-\Delta_{\ell} u=g \quad \text { on } \partial^{\prime \prime} Q_{T}, \quad u(x, 0)=0 \quad \text { on } \bar{\Omega} .
$$

Here the equation in $Q_{T}$ and the equation on $\partial^{\prime \prime} Q_{T}$ are decoupled. So we can first solve the boundary equation and then use its solution as the Dirichlet datum for the equation in the domain. The estimates similar to Theorem 2.1, combined with Proposition 1.1, show that the solution belongs to $V_{\sigma}^{2,1}\left(Q_{T}, \partial^{\prime \prime} Q_{T}\right)$ and inequality (3.2) holds. So the claim follows.

The estimates in Theorem 2.1 show that the operator

$$
\mathcal{L}_{\mu}-\mathcal{L}_{0}: V_{\sigma}^{2,1}\left(Q_{T}, \partial^{\prime \prime} Q_{T}\right) \rightarrow L_{\sigma}^{2}\left(Q_{T}\right) \times L^{2}\left(\partial^{\prime \prime} Q_{T}\right) ; \quad \mathcal{L}_{\mu} u-\mathcal{L}_{0} u=\mu\left(a u, \frac{\partial u}{\partial \nu}+b u+\theta_{s}(u)\right)
$$

is compact. Since $\operatorname{Ker}\left(\mathcal{L}_{1}\right)$ is trivial by Lemma 3.1, the operator $\mathcal{L}_{1}$ is also invertible, and the proof is complete. 
If $\Omega$ is a convex polygon, then $\alpha<\pi$. Hence, we can choose $\sigma=0$ and we obtain the following result.

Corollary 3.3. Let $\Omega$ be a convex polygon. Suppose that $f \in L^{2}\left(Q_{T}\right), g \in L^{2}\left(\partial^{\prime \prime} Q_{T}\right)$, $a \in L^{\infty}\left(Q_{T}\right)$ and $b \in L^{\infty}\left(\partial^{\prime \prime} Q_{T}\right)$. Then the problem (1.1)-(1.3) has a unique solution $u \in$ $H^{2,1}\left(Q_{T}\right) \cap H^{2,1}\left(\partial^{\prime \prime} Q_{T}\right)$, and the following inequality holds:

$$
\|u\|_{H^{2,1}\left(Q_{T}\right)}^{2}+\|u\|_{H^{2,1}\left(\partial^{\prime \prime} Q_{T}\right)}^{2} \leq C\left(\|f\|_{L^{2}\left(Q_{T}\right)}^{2}+\|g\|_{L^{2}\left(\partial^{\prime \prime} Q_{T}\right)}^{2}\right)
$$

where $C$ depends on $T, a$ and $b$.

If $\Omega$ is not convex, then $\pi<\alpha<2 \pi$. In this case the solution in general does not belong to $H^{2,1}\left(Q_{T}\right)$ even for $f=0$, see e.g. [18] for the asymptotics of solution to the Dirichlet problem.

Remark 3.4. In [10] we considered the elliptic case, in particular we proved that the solution of the elliptic problem belongs to $H^{2}(\partial \Omega)$. Actually, similarly to Proposition 1.1, we have that $u \in \tilde{H}^{2}(\partial \Omega)$. In turn, this implies that the hypothesis $s<\frac{3}{4}$ is not needed in [10, Theorem 2.1].

Remark 3.5. All our results easily hold for an arbitrary piecewise smooth domain $\Omega \subset \mathbb{R}^{2}$ without cusps.

\section{A Appendix. The extension theorem}

Theorem A.1. Let $u \in H^{2,1}\left(\partial^{\prime \prime} Q_{T}\right)$ and $\left.u\right|_{t=0}=0$. Then there exists an extension $U \in$ $H^{\frac{5}{2}, \frac{5}{4}}\left(\mathbb{R}^{2} \times \mathbb{R}\right)$ such that $\left.U\right|_{t=0}=0,\left.U\right|_{\partial^{\prime \prime} Q_{T}}=u$, and

$$
\|U\|_{H^{\frac{5}{2}, \frac{5}{4}\left(\mathbb{R}^{2} \times \mathbb{R}\right)}} \leq C\|u\|_{H^{2,1}\left(\partial^{\prime \prime} Q_{T}\right)} .
$$

Proof. By localization, we can consider separately the extension from a face $l_{j} \times(0, T)$ and the extension from a neighborhood of a corner. Using standard extension from a face to the plane containing this face

$$
H^{2,1}\left(l_{j} \times(0, T)\right) \rightarrow H^{2,1}(\mathbb{R} \times \mathbb{R})
$$

we reduce the first operation to the extension from a plane and the second one to the extension from a pair of half-planes intersecting on the $t$-axis. Using a proper linear coordinates transform, we can assume that these half-planes are orthogonal. Since $\left.u\right|_{t=0}=0$, we can 
suppose without loss of generality that the extended function is odd w.r.t. $t$. Moreover, in what follows all extensions are supposed compactly supported.

We now denote

$$
\begin{gathered}
\Pi^{1}=\left\{\left(x_{1}, x_{2}, t\right) \in \mathbb{R}^{2} \times \mathbb{R}: x_{2}=0\right\} ; \quad \Pi^{2}=\left\{\left(x_{1}, x_{2}, t\right) \in \mathbb{R}^{2} \times \mathbb{R}: x_{1}=0\right\} ; \\
\Pi_{ \pm}^{j}=\left\{\left(x_{1}, x_{2}, t\right) \in \Pi^{j}: x_{j} \gtrless 0\right\}, \quad j=1,2 .
\end{gathered}
$$

We introduce a mollifier $\phi\left(x_{1}, t\right) \in \mathcal{C}_{0}^{\infty}(\mathbb{R} \times \mathbb{R})$ such that $\phi$ is radially symmetric, $\operatorname{supp}(\phi) \subset$ $B_{1}(0)$, and $\int_{\mathbb{R} \times \mathbb{R}} \phi d x_{1} d t=1$. The extension from the plane $\Pi_{1}$ is defined in a standard way [27] via the $2 \mathrm{D}$ Fourier transform (here $\left(\xi_{1}, \tau\right)$ are the variables dual to $\left.\left(x_{1}, t\right)\right)$ :

$$
\widehat{U}\left(\xi_{1}, x_{2}, \tau\right)=\left(\widehat{\mathcal{P}_{1} u}\right)\left(\xi_{1}, x_{2}, \tau\right):=\widehat{\phi}\left(\left(1+\xi_{1}^{4}+\tau^{2}\right)^{\frac{1}{4}}\left|x_{2}\right|\right) \cdot \widehat{u}\left(\xi_{1}, \tau\right) .
$$

Direct (and standard) calculation using the Parseval identity provides the estimate (A.1). Moreover, since $u$ is odd w.r.t. $t, \mathcal{P}_{1} u$ is also odd w.r.t. $t$. In the same way we define the extension operator $\mathcal{P}_{2}$ from the plane $\Pi_{2}$.

To manage the extension from $\Pi_{+}^{1} \cup \Pi_{+}^{2}$, we first extend

$$
H^{2,1}\left(\Pi_{+}^{1}\right) \cap H^{2,1}\left(\Pi_{+}^{2}\right) \rightarrow H^{2,1}\left(\Pi^{1}\right) \cap H^{2,1}\left(\Pi^{2}\right)
$$

and we apply the operator $\mathcal{P}_{2}$ to $\left.u\right|_{\Pi^{2}}$. It remains to extend the function

$$
v=\left(u-\mathcal{P}_{2}\left(\left.u\right|_{\Pi^{2}}\right)\right)_{\Pi_{1}}
$$

so that the extension $V$ vanishes on $\Pi_{+}^{2}$.

We split $v$ into the $\operatorname{sum} v=v_{0}+v_{+}+v_{-}$, where

$$
v_{0}\left(x_{1}, t\right)=\frac{v\left(x_{1}, t\right)-v\left(-x_{1}, t\right)}{2} \quad \text { and } \quad v_{ \pm}\left(x_{1}, t\right)=\frac{v\left(x_{1}, t\right)+v\left(-x_{1}, t\right)}{2} \cdot \chi\left( \pm x_{1}\right)
$$

( $\chi$ stands for the Heaviside function). Since $v_{0}$ is odd w.r.t. $x_{1}$, the function $V_{0}=\mathcal{P}_{1} v_{0}$ is also odd w.r.t. $x_{1}$ and thus vanishes on $\Pi_{+}^{2}$.

Next, we notice that the function $v\left(x_{1}, t\right)+v\left(-x_{1}, t\right)$ is even w.r.t. $x_{1}$ and vanishes on the line $x_{1}=0$. Therefore, $v_{ \pm} \in H^{2,1}\left(\Pi^{1}\right)$.

Since $v_{+}$is supported in $\Pi_{+}^{1}$, we immediately obtain that the support of the function $\mathcal{P}_{1} v_{+}$lies in the wedge $x_{1} \geq-\left|x_{2}\right|$. Hence, the function

$$
V_{+}\left(x_{1}, x_{2}, t\right)=\left(\mathcal{P}_{1} v_{+}\right)\left(x_{1}-x_{2}, x_{2}, t\right)
$$

is an extension of $v_{+}$having the required smoothness and vanishing on $\Pi_{+}^{2}$. In a similar way, we define

$$
V_{-}\left(x_{1}, x_{2}, t\right)=\left(\mathcal{P}_{1} v_{-}\right)\left(x_{1}+x_{2}, x_{2}, t\right) .
$$

Setting $V=V_{0}+V_{+}+V_{-}$, the thesis follows. 
Acknowledgements. S. C. and M. R. L. have been supported by the Gruppo Nazionale per l'Analisi Matematica, la Probabilità e le loro Applicazioni (GNAMPA) of the Istituto Nazionale di Alta Matematica (INdAM). A. N. was partially supported by Russian Foundation for Basic Research grant 18-01-00472. M. R. L. would like to acknowledge networking support by the COST Action CA18232.

This work was undertaken while A. N. was visiting the department of Basic and Applied Sciences for Engineering of Sapienza Università di Roma supported by St. Petersburg University (project 41126689) and by the international agreement between Sapienza Università di Roma and Steklov Mathematical Institute of Russian Academy of Sciences.

We are thankful to Professor Vladimir Gol'dshtein (Ben-Gurion University) for the hint to the proof of Theorem A.1 and to Professor Mikhail Surnachev (Keldysh Institute of Applied Mathematics) for some important comments.

\section{References}

[1] D. E. Apushkinskaya, A. I. Nazarov, A survey of results on nonlinear Venttsel' problems, Appl. Math., 45 (2000), no. 1, 69-80.

[2] D. E. Apushkinskaya, A. I. Nazarov, Quasilinear two-phase Venttsel' problems, ZNS POMI, 271 (2000), 11-38 (Russian); English transl.: J. Math. Sci., 115 (2003), N6, 2704-2719.

[3] D. E. Apushkinskaya, A. I. Nazarov, Linear two-phase Venttsel' problems, Ark. Mat., 39 (2001), no. 2, 201-222.

[4] D. E. Apushkinskaya, A. I. Nazarov, D. K. Palagachev, L. G. Softova, Venttsel boundary value problems with discontinuous data, preprint available at https://arxiv.org/abs/1907.03017. 32p.

[5] W. Arendt, G. Metafune, D. Pallara, S. Romanelli, The Laplacian with Wentzell-Robin boundary conditions on spaces of continuous functions, Semigroup Forum, 67 (2003), no. 2, 247-261.

[6] F. Brezzi, G. Gilardi, Fundamentals of PDE for Numerical Analysis, in: Finite Element Handbook, McGraw-Hill Book Co., New York, 1987.

[7] M. Cefalo, S. Creo, M. R. Lancia, P. Vernole, Nonlocal Venttsel' diffusion in fractal-type domains: regularity results and numerical approximation, Math. Methods Appl. Sci., 42 (2019), no. $14,4712-4733$.

[8] M. Cefalo, G. Dell'Acqua, M. R. Lancia, Numerical approximation of transmission problems across Koch-type highly conductive layers, Appl. Math. Comput., 218 (2012), no. 9, 5453-5473. 
[9] M. Cefalo, M. R. Lancia, H. Liang, Heat-flow problems across fractals mixtures: regularity results of the solutions and numerical approximations, Differential Integral Equations, 26 (2013), no. 9-10, 1027-1054.

[10] S. Creo, M. R. Lancia, A. I. Nazarov, P. Vernole, On two-dimensional nonlocal Venttsel' problems in piecewise smooth domains, Discrete Contin. Dyn. Syst. Series S, 12 (2019), no. 1, 57-64.

[11] S. Creo, M. R. Lancia, A. Vélez-Santiago, P. Vernole, Approximation of a nonlinear fractal energy functional on varying Hilbert spaces, Commun. Pure Appl. Anal., 17 (2018), no. 2, 647-669.

[12] S. Creo, M. R. Lancia, P. Vernole, Convergence of fractional diffusion processes in extension domains, J. Evol. Equ., 20 (2020), no. 1, 109-139.

[13] S. Creo, V. Regis Durante, Convergence and density results for parabolic quasi-linear Venttsel' problems in fractal domains, Discrete Contin. Dyn. Syst. Series S, 12 (2019), no. 1, 65-90.

[14] G. Goldstein Ruiz, Derivation and physical interpretation of general boundary conditions, Adv. Differential Equations, 11 (2006), no. 4, 457-480.

[15] V. P. Il'in, Some integral inequalities and their applications in the theory of differentiable functions of several variables, Mat. Sb. (N.S.), 54(96) (1961), no. 3, 331-380 (Russian).

[16] V. A. Kondrat'ev, Boundary-value problems for elliptic equations in domains with conical or angular point, Trans. Moscow Math. Soc., 16 (1967), 209-292.

[17] V. A. Kozlov, Coefficients in the asymptotics of the solutions of initial-boundary value parabolic problems in domains with a conic point, Siberian Math. J., 29 (1988), no. 2, 222-233.

[18] V. A. Kozlov, V. G. Maz'ya, Singularities of solutions of the first boundary value problem for the heat equation in domains with conical points. II, Soviet Math. (Iz. VUZ), 31 (1987), no. $3,61-74$.

[19] O. A. Ladyzhenskaya, The Boundary Value Problems of Mathematical Physics, Applied Mathematical Sciences, 49, Springer-Verlag, New York, 1985.

[20] M. R. Lancia, V. Regis Durante, P. Vernole, Asymptotics for Venttsel' problems for operators in non divergence form in irregular domains, Discrete Contin. Dyn. Syst. Ser. S, 9 (2016), no. $5,1493-1520$. 
[21] M. R. Lancia, A. Vélez-Santiago, P. Vernole, Quasi-linear Venttsel' problems with nonlocal boundary conditions on fractal domains, Nonlinear Anal. Real World Appl., 35 (2017), 265291.

[22] M. R. Lancia, A. Vélez-Santiago, P. Vernole, A quasi-linear nonlocal Venttsel' problem of Ambrosetti-Prodi type on fractal domains, Discrete Cont. Dyn. Syst., 39 (2019), no. 8, 44874518.

[23] M. R. Lancia, P. Vernole, Venttsel' problems in fractal domains, J. Evol. Equ., 14 (2014), no. $3,681-712$.

[24] J. L. Lions, E. Magenes, Non-Homogeneous Boundary Value Problems and Applications, Vol. I, Springer-Verlag, New York-Heidelberg, 1972.

[25] A. I. Nazarov, On the nonstationary two-phase Venttsel problem in the transversal case, J. Math. Sci. (N. Y.), 122 (2004), no. 3, 3251-3264.

[26] J. Necas, Les Mèthodes Directes en Thèorie des Èquationes Elliptiques, Masson, Paris, 1967.

[27] L. N. Slobodetskii, Generalized S. L. Sobolev spaces and their application to the boundary value problems for partial differential equations, Scientific Notes of Leningrad State Pedagogical Institute, 197 (1958), 54-112 (Russian).

[28] A. Vélez-Santiago, Quasi-linear variable exponent boundary value problems with WentzellRobin and Wentzell boundary conditions, J. Funct. Anal., 266 (2014), no. 2, 560-615.

[29] A. Vélez-Santiago, Global regularity for a class of quasi-linear local and nonlocal elliptic equations on extension domains, J. Funct. Anal., 269 (2015), no. 1, 1-46.

[30] A. D. Venttsel', On boundary conditions for multidimensional diffusion processes, Theor. Probability Appl., 4 (1959), no. 2, 164-177.

[31] M. Warma, An ultracontractivity property for semigroups generated by the p-Laplacian with nonlinear Wentzell-Robin boundary conditions, Adv. Differential Equations, 14 (2009), no. 7-8, 771-800.

[32] M. Warma, The p-Laplace operator with the nonlocal Robin boundary conditions on arbitrary open sets, Annali Mat. Pura Appl. (4), 193 (2014), no. 1, 203-235. 